\title{
REPERCUSSÕES HEMODINÂMICAS NOS PACIENTES TRANSPORTADOS POR HELICÓPTERO NO OESTE CATARINENSE
}

Augusto Bortolanza DALAZEN¹; Guilherme Caio UEZ²; Rogério de Souza BARCALA ${ }^{3}$;

\section{RESUMO}

O SAER-Fron/SARA atua desde 2015 em Chapecó-SC, como órgão de assistência aeromédica, atendendo e transferindo doentes. Verificar as repercussões hemodinâmicas dos pacientes após o voo, ajuda a avaliar se este, influencia na homeostase das vítimas transportadas e assim ratificar o helicóptero como um método seguro. Metodologia: Trata-se de um estudo observacional, descritivo, transversal. Foram analisados prontuários de pacientes transportados entre agosto de 2018 a janeiro de 2019. As análises estatísticas foram efetuadas no software Statisctical Packet for Social Sciences (20.0), tendo 95\% de nível de confiança e 5\% de erro amostral. O comitê de ética em pesquisa da Unochapecó aprovou o projeto sob parecer 2.799.088. Seu desenvolvimento ocorreu conforme resolução CNS 466/12. Resultados: Houve 67 missões, com 39 pacientes masculinos, 27 femininos e em uma ocorrência não estava especificado o sexo. Quanto aos fatores hemodinâmicos, em relação a pressão arterial média (PAM) antes ao voo, teve-se um aumento de 0,595 $\mathrm{mmHg}$ da PAM após o voo, além disso, quando ao batimento do coração antes do voo, apresentou uma predição de redução da PAM após voo de $0,036 \mathrm{mmHg}$. Somado a isso, a saturação periférica de oxigênio $\left(\mathrm{SpO}_{2}\right)$ antes o voo, correlacionou-se com um aumento de $0,303 \%$ na $\mathrm{SpO}_{2}$ final e para o batimento do coração antes do voo, apresentou uma redução de $0,005 \%$ na $\mathrm{SpO}_{2}$ final. Conclusão: Nota-se pequena variação dos fatores hemodinâmicos após o voo, sugerindo baixa repercussão do voo no organismo da vítima e uma boa estabilização pelos membros da equipe de resgate.

Palavras-chave: Hemodinâmica, Serviços médicos de emergência, Resgate aéreo.

\section{INTRODUÇÃO}

Ao longo de um voo de helicóptero, os passageiros a bordo, estão expostos a fatores estressores, entre eles, destacam-se ambientais e físicos, cujos acometimentos influem de forma direta no trabalho de atendimento da tripulação. Como estressores físicos e ambientais devemos considerar a hipóxia em função da altitude, o clima, a temperatura, o vento, o tempo da missão, o tipo de voo e áreas de pouso, o ruído, e vibrações da aeronave (CARCHIETTI et al., 2011).

A pressão atmosférica está diretamente ligada a quadros de queda dos níveis de oxigênio, devido a redução pressórica de forma não linear com a altitude. Tal decréscimo representa uma redução na pressão alveolar de oxigênio, ocasionando o quadro levando a diversas manifestações, como taquicardia, bradicardia, arritmias, hipotensão, distúrbios taquipneicos e alteração dos níveis de consciência (BEARD; LAX; TINDALL, 2016). 


\section{CONAER

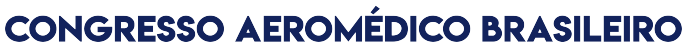

Além da hipóxia, sabe-se que quanto maior a altitude, o volume de qualquer gás confinado aumentará. Conforme a Lei de Boyle, um volume de gás é inversamente proporcional à pressão que o rodeia. Assim sendo, qualquer cavidade corpórea repleta de gás sofrerá interferência. Os locais mais comuns de distúrbios gasosos no paciente típico envolvem os ouvidos, seios da face e órgãos do trato gastrointestinal (TGI) (HURD; JERNIGAN, 2002; BEARD; LAX; TINDALL, 2016). A hipóxia hipobárica em um quadro cardiovascular também deve ser levada em consideração. Cardiopatas a compensam até certo ponto aumentando a ventilação, inicialmente ocorre uma leve taquicardia, o que faz com que o miocárdio demande mais oxigênio. Naqueles com reserva cardíaca limitada, a diminuição da oferta de oxigênio e a taquicardia reflexa pode levar à descompensação cardíaca. Vale destacar que em voos comerciais, pessoas com angina instável têm contraindicação clara ao voo. Assim como, pacientes com infarto agudo do miocárdio (IAM) recente não complicado não devem voar até 2-3 semanas após o evento e estando de volta às atividades diárias (AEROSPACE MEDICAL ASSOCIATION MEDICAL GUIDELINES TASK FORCE, 2003).

Em relação à temperatura ambiental, há uma diminuição de $2^{\circ} \mathrm{C}$ por cada 300 metros (aproximadamente 1000 pés) de altitude, em aeronaves não pressurizadas. Há ainda as vibrações, cujo principal efeito é interferir no sinal de monitores e contadores das bombas de infusão contínua, necessitando de reprogramação periódica (RAJDL, 2011).

Desse modo, verificar a influência das condições sob as quais o paciente é exposto ao ser transportado por helicóptero e suas repercussões em seus parâmetros hemodinâmicos (frequência cardíaca, pressão arterial média e saturação periférica de oxigênio), auxilia na criação de medidas que visam a menor interferência do meio na vítima, ou confirmar, como um método de resgate seguro.

\section{METODOLOGIA}

Trata-se de um estudo observacional, descritivo do tipo transversal. Foram analisados os prontuários de todos os pacientes transportados, no período de agosto de 2018 a janeiro de 2019. Foram excluídos os prontuários de pacientes que estavam em óbito na chegada da equipe ou que foram a óbito antes do transporte.

As análises estatísticas foram realizadas por meio do software Statisctical Packet for Social Sciences (SPSS) versão 20.0. Para as variáveis qualitativas foram 
realizadas análise de frequência (absoluta) e para as variáveis quantitativas foram realizadas análises de tendência central (média; desvio padrão; mediana; intervalo interquartil; mínimo; e máximo). A fim de relacionar a média das variações dos fatores hemodinâmicos (pressão arterial média, frequência cardíaca e saturação periférica de oxigênio), foi realizada regressão linear multivariada. Todas as análises estatísticas levaram em consideração o nível de confiança de 95\% e erro amostral de $5 \%$.

O projeto que deu origem ao estudo foi aprovado pelo Comitê de Ética em Pesquisa (CEP) da Unochapecó, conforme consta no parecer 2.799.088 emitido por este comitê. O desenvolvimento deste estudo se deu conforme resolução CNS $466 / 12$.

\section{RESULTADOS E DISCUSSÕES}

67 pacientes foram transportados no período, 39 do sexo masculino e 27 do sexo feminino, sendo que em uma ocorrência não havia sido registrado o sexo no prontuário.

As alterações sofridas por um organismo durante o voo podem ser diversas, porém, parâmetros hemodinâmicos como frequência cardíaca (FC), pressão arterial (PA) e saturação periférica de oxigênio $\left(\mathrm{SpO}_{2}\right)$ são as que mais refletem o real estresse das missões. Os resultados desses parâmetros encontrados na pesquisa, estão sumarizados na tabela 1.

\begin{tabular}{|c|c|c|c|c|c|c|}
\hline \multicolumn{7}{|c|}{ Variáveis dependentes } \\
\hline \multirow[t]{2}{*}{$\begin{array}{c}\text { Variáveis } \\
\text { independentes }\end{array}$} & \multicolumn{2}{|c|}{$\begin{array}{c}\text { PAM } \\
\text { depois do voo }\end{array}$} & \multicolumn{2}{|c|}{$\begin{array}{c}\mathrm{SpO}_{2} \\
\text { depois do voo }\end{array}$} & \multicolumn{2}{|c|}{$\begin{array}{c}\text { FC } \\
\text { depois do voo }\end{array}$} \\
\hline & Beta & $P$ & Beta & $P$ & Beta & $P$ \\
\hline & 170,934 & 017 & 68,947 &, 000 & 110,755 & 398 \\
\hline $\begin{array}{l}\text { PAM antes do voo } \\
\text { (mmHg) }\end{array}$ &, 595 & ${ }^{*}<0,001$ &,- 019 & ,329 & ,122 & ,644 \\
\hline $\begin{array}{l}\mathrm{SpO}_{2} \text { antes do } \\
\text { voo }(\%)\end{array}$ & $-1,098$ & ,112 & ,303 & ${ }^{*}, 006$ &,- 623 & ,636 \\
\hline $\begin{array}{l}\text { FC antes do voo } \\
\text { (bpm) }\end{array}$ &,- 036 & ${ }^{*}, 002$ &,- 005 & ${ }^{*}, 004$ & ,037 & ,076 \\
\hline $\begin{array}{l}{ }^{*} E C G \text { antes do } \\
\text { voo }\end{array}$ & ,597 & ,539 & ,002 & ,990 &,- 101 & ,958 \\
\hline $\begin{array}{l}\text { Tempo de voo } \\
\text { (min) }\end{array}$ & ,378 & ,300 &,- 018 & ,731 & ,953 & ,191 \\
\hline $\begin{array}{l}\text { Distância do voo } \\
(\mathrm{km})\end{array}$ &,- 005 & 1,000 & ,004 & ,818 &,- 286 & , 188 \\
\hline Altitude $(\mathrm{m})$ &,- 041 & ,127 & ,002 &, 546 & ,014 & ,781 \\
\hline
\end{tabular}

\footnotetext{
${ }^{*} P$ estatisticamente significativos. ${ }^{* \star} \mathrm{ECG}=$ escala de coma de Glasgow.
} 


\section{CONAER

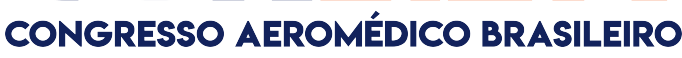

Com base a tabela, a pressão arterial média (PAM), correlacionou-se com um aumento de 0,595 mmHg após o voo ( $\mathrm{p}<0,001)$, ademais, para a FC, associou-se a uma predição de redução da PAM de $0,036 \mathrm{mmHg}(p<0,002)$ e uma redução de $0,005 \%$ na $\mathrm{SpO}_{2}$ final $(\mathrm{p}<0,004)$. Somado a esses dados, há que a $\mathrm{SpO}_{2}$ se correlacionou com um aumento de $0,303 \%$ após o transporte $(p<0,006)$. Referente aos demais resultados, não houve significância estatística.

Em comparação a Carchietti et al. (2011), que tabelou as variações hemodinâmicas nos membros da equipe de resgate, denota que, na análise multivariada dos parâmetros após o voo, nenhuma das características pareceu influenciar significativamente os parâmetros vitais no final da missão, e quando se analisou apenas $01^{\circ}$ voo de cada turno de trabalho, também não surgiram diferenças significativas. É importante notar que, neste estudo, as variações foram analisadas nos membros da equipe de resgate e não nos pacientes transportados.

\section{CONSIDERAÇÕES FINAIS}

Quanto a influência do voo nos fatores hemodinâmicos dos pacientes, não se encontrou relação estatisticamente significante entre as variáveis impostas pelo helicóptero (altitude, velocidade, temperatura, vibração e tempo de voo) e os fatores do paciente (PA, FC e $\mathrm{SpO}_{2}$ ). Contudo, houve correlação entre alguns parâmetros da vítima antes e após o voo, mesmo pequenos, estatisticamente relevantes. Desse modo é possível notar que essa pequena variação dos fatores hemodinâmicos após o voo, sugerem baixa repercussão do voo no organismo da vítima transportada e uma boa estabilização da vítima através dos tratamentos impostos pelos membros da equipe de resgate.

\section{REFERÊNCIAS}

Aerospace Medical Association Medical Guidelines Task Force. Medical Guidelines for Airline Travel 2nd Edition. Aviation, Space, and Environmental Medicine, v. 74, n. 5, maio 2003.

BEARD, Laura; LAX, Peter; TINDALL, Marcus. Physiological effects of transfer for critically ill patients. World Federation of Societies of Anesthesiologists ATOTW, [s.I.] n. 330, maio 2016.

CARCHIETTI, Elio et al. Influence of Stressors on HEMS Crewmembers in Flight. Air Medical Journal, [s.I.], v. 30, n. 5, 2011.

HURD, William W.; JERNIGAN, John G. Aeromedical Evacuation Management of Acute and Stabilized Patients. Springer, 2002.

RAJDL, E. Aerotransporte: Aspectos Básicos y Clínicos. Revista Médica Clínica Las Condes, v. 22, n. 3, p. 389-96, 2011. 